\title{
Hematoma volume and other prognostic factors with mortality in spontaneous intracerebral hemorrhage
}

\author{
HB Rotzel", A Serrano Lázaro, D Aguillón Prada, A Mesejo Arizmendi, C Sanchís Piqueras, M Rodriguez Gimillo, \\ ML Blasco Cortés, MN Carbonell Monleón, O Díaz Barina
}

From ESICM LIVES 2015

Berlin, Germany. 3-7 October 2015

\section{Introduction}

Intracerebral hemorrhage ( $\mathrm{ICH}$ ), a subtype of stroke associated with high mortality and the majority of survivors will remain permanently disabled. Identifying prognostic factors will determine the severity and guide the treatment for different patients.

\section{Objective}

To relate the volume of hematoma and other prognostic factors with mortality in patients with spontaneous intracerebral hemorrhage (ICH).

\section{Material and Methods}

All patients admitted to the ICU with the diagnosis of spontaneous ICH were collected. We determine the hematoma volume at admission with the modified formula Kothari $(\mathrm{AxBxC} / 2 \mathrm{cc})$, the location of hemastom as posterior fossa or supratentorial, severity scales (GCS at admission, SOFA, APACHE II), medical history and complications during the ICU admission. Used percentils $(\%$,$) mean (SD) and median (min / max). For the$ univariate analysis we used U-Mann Whitney and Chi square with $\mathrm{p}<0.05$ significant. Subsequently we performed a multivariate analysis using binary regression (OR with 95\% CI) and significant $\mathrm{p}<0.05$.

\section{Results}

101 patients were included. $66 \%$ men with a mean age $61.8 \pm 12.7$ years. Our overall mortality was $35 \%$. 82\% were supratentorial and $16 \%$ infratentorial. The median APACHE II 13 (0-30), SOFA 4 (0-14), GCS 12 (3-15) and mean volume of $37.8 \mathrm{cc}$ hematoma. In the univariate analysis was significantly correlated with mortality: GCS at admission (p 0.001), APACHE II (p 0.001) and
SOFA (p 0.000), taking oral anticoagulants (ACOs) (p 0.05 ), LOS in hospital ( $\mathrm{p} 0.05$ ) and complications as intracranial hypertension ( $\mathrm{p} 0.000$ ), rebleeding (0.01), herniation ( $p 0.000)$ and eadema $( \pm 0,001)$. After the multivariate analysis, only de volume of hematoma (OR 1.2, 95\% CI 1.1-1.2; p 0.01), intracranial hypertension in the first 48 h (OR 35 ; 95\% CI 1.12 to 10.99; p 0.03), herniation (OR 7.08, 95\% CI 2.77 to 18.09; p 0.000) and SOFA (OR 1.35; $95 \% 1.16$ to 1.57 ; p 0.000 ). was correlated significant with mortality. As for the volume of hematoma we determied a cuttoff point $>20 \mathrm{cc}$ obtaining statistical significance in its relation with mortality (p 0.02. In a subgroup analysis between posterior fossa and supratentorial with volumes $>15 \mathrm{cc}$ and $45 \mathrm{cc}$ respectively we obtained also statistically significance with mortality (p 0.037 and 0.038 respectively).

\section{Conclusions}

SOFA, volumen of hematoma at admission, intracranial hypertension and herniation are related to mortality in patients with spontaneous intracerebral hemorrhage.

Published: 1 October 2015

\section{References}

1. Specogna AV, Turin TC, Patten SB, Hill MD: Factors associated with early deterioration after spontaneous intracerebral hemorrhage: a systematic review and meta-analysis. PLoS One 2014, 9(5):e96743, May 8; doi:10.1371/ journal.pone.0096743. eCollection 2014.

2. Gupta M, Verma R, Parihar A, Garg RK, Singh MK, Malhotra HS: Perihematomal edema as predictor of outcome in spontaneous intracerebral hemorrhage. J Neurosci Rural Pract 2014, 5(1):48-54, Jan; doi: 10.4103/0976-3147.127873.

doi:10.1186/2197-425X-3-S1-A981

Cite this article as: Rotzel et al.: Hematoma volume and other prognostic factors with mortality in spontaneous intracerebral hemorrhage. Intensive Care Medicine Experimental 2015 3(Suppl 1):A981.

Hospital Clinic Universitario de Valencia, Valencia, Spain 